\title{
First Case of Coloboma, Lens Neovascularization, Traumatic Cataract and Retinal Detachment in a Young Asian Female
}

\author{
Bharat Gurnani ${ }^{1}$, Kirandeep Kaur ${ }^{2}$, and Subashini Sekaran ${ }^{3}$ \\ ${ }^{1}$ Aravind Eye Care System \\ ${ }^{2}$ Aravind Eye Hospital, Pondicherry \\ ${ }^{3}$ Aravind Eye Hospital, Pondicherry, India
}

June 29, 2021

\begin{abstract}
A young girl post-stick injury presented with traumatic cataract and lens neovascularization in OS and iris coloboma, key whole pupil, and cataractous changes in OD. Fundoscopy revealed chorioretinal coloboma OD and retinal detachment on B scan OS. In view of guarded visual prognosis, the patient was advised observation in $\mathrm{OU}$
\end{abstract}

First Case of Coloboma, Lens Neovascularization, Traumatic Cataract and Retinal Detachment in a Young Asian Female

\begin{abstract}
A young girl post stick injury presented with traumatic cataract and lens neovascularization in OS and iris coloboma, key whole pupil and cataractous changes in OD. Fundoscopy revealed chorioretinal coloboma OD and retinal detachment on B scan OS. In view of guarded visual prognosis, the patient was advised observation in $\mathrm{OU}$.
\end{abstract}

\section{Case description}

A 25 years old girl presented with recent onset deterioration of vision in the left eye (OS) in our OPD. She gave the history of trauma with the stick in OS 2 months back. Best Corrected Visual Acuity (BCVA) was hand movements in the right eye (OD) and no Perception of light (No PL) in OS. Intraocular pressure was $14 \mathrm{mmHg}$ OD and $4 \mathrm{mmHg}$ OS. Detailed anterior segment examination in OD revealed horizontal jerky nystagmus, inferior iris coloboma, keyhole pupil, and focal cataractous changes (Figure 1a). Ocular examination of OS revealed horizontal jerky nystagmus, circumciliary congestion, shallow anterior chamber, multiple sphinter tears with atrophic iris, irregular pupil, and cataractous lens with numerous discrete blood vessels over the lens capsule (Figure 1b). Fundoscopy in OD revealed chorioretinal coloboma involving the disc and macula (Figure 1c) and was obscured in OS secondary to traumatic cataract. Ultrasound B scan depicted lens echoes and moderate to high reflective membranous echoes attached to optic nerve head posteriorly and posterior aspect of lens anteriorly. The membranous echoes in the posterior segment showed limited mobility (Figure 1d). A diagnosis of iris coloboma, focal cataract, and chorioretinal coloboma was made in OD, and iris coloboma, lens neovascularization, traumatic cataract, and retinal detachment were made in OS. This is a very rare and probably the first case of coloboma, cataract, retinal detachment, and lens neovascularization. Surgical intervention was not considered in either eye in view of poor visual prognosis.

Ocular trauma is one of the major yet unrecognized causes of vision loss globally. The complications of blunt ocular trauma like corneal edema, keratitis, hyphema, secondary glaucoma, cataract, and retinal detachment 
are well known. ${ }^{1}$. Neovascularization has been reported in various ocular structures like cornea, iris, anterior chamber angle, retina, and rarely in the lenticular stroma. But lens neovascularization has rarely been reported. ${ }^{2}$

\section{Ethics statement}

Written informed consent for publication of photographs was obtained from the patient. This case report did not receive any funding. Authors have access to all source data for this case report.

\section{Acknowledgements}

None.

\section{Conflict of interest}

None.

\section{Author contributions}

BG has made substantial contributions in the acquisition and interpretation of data, analysis drafting and revision of the manuscript. KK has made substantial contributions in the analysis and interpretation of the data, and in revising the manuscript. SS helped in data collection of the patient. All authors read and approved the final version of the manuscript and agree to be accountable for all aspects of the work.

\section{References}

1.Cassen JH. Ocular trauma. Hawaii Med J. 1997 Oct;56(10):292-4. PMID: 9385749.

2. Kabat AG. Lenticular neovascularization subsequent to traumatic cataract formation. Optom Vis Sci. 2011 Sep;88(9):1127-32. doi: 10.1097/OPX.0b013e31822311e2. PMID: 21642887.

Figure legends

Figure 1a- Image of the right eye depicting inferior iris coloboma, key whole pupil and focal cataractous changes

Figure 1b - Image of the left eye depicting inferior iris coloboma with multiple sphinter tear and patches of iris atrophy, key hole pupil and a vascularized membrane in the anterior chamber

Figure 1c- Fundus image of the right eye depicting chorioretinal coloboma involving the disc and macula

Figure 1d - B scan image of the left eye depicting lens echoes and moderate to high reflective membranous echoes attached to optic nerve head posteriorly and posterior aspect of lens anteriorly 


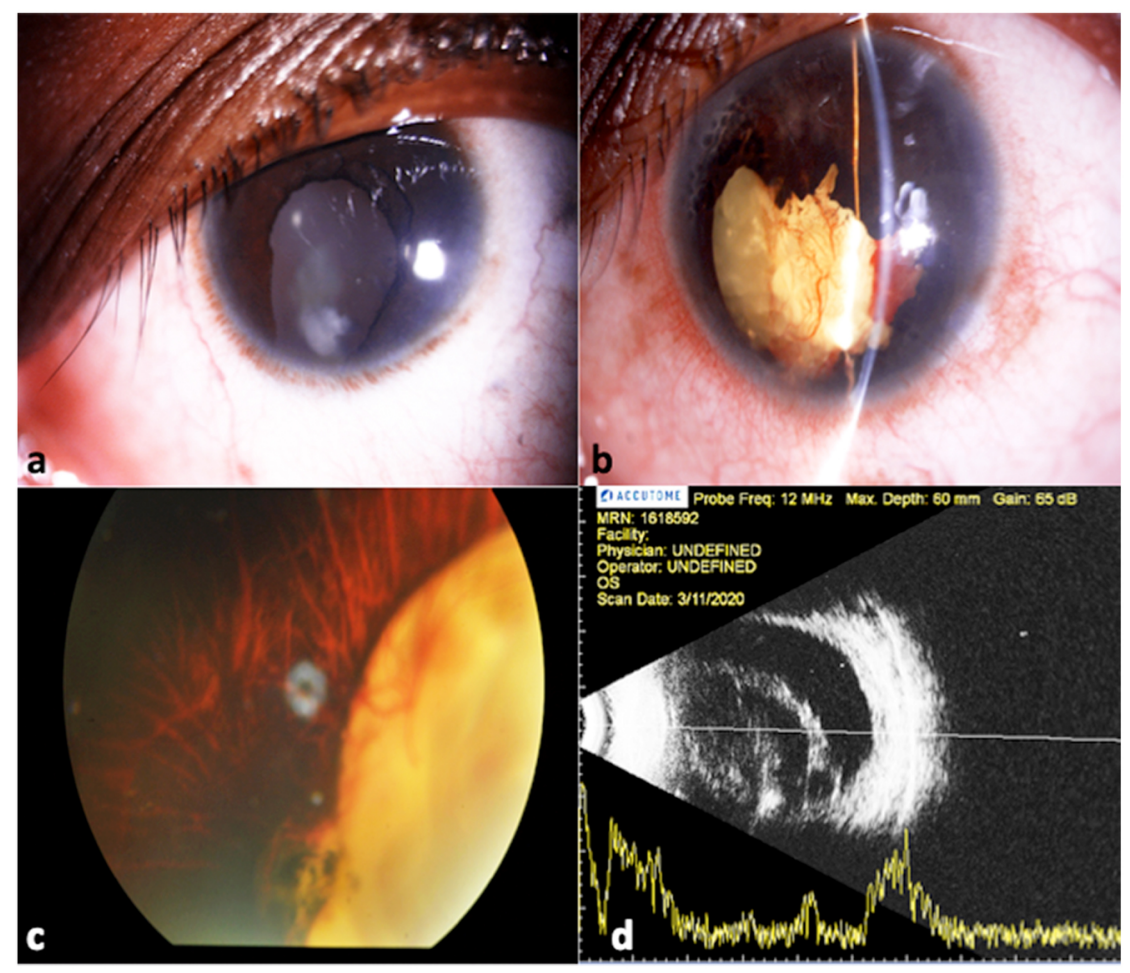

